\title{
Incidencia de la gestión por competencias en el crecimiento empresarial
}

\section{Impact of management by competencies in business growth}

Stefany Elizabeth Correa Jiménez

Universidad Técnica de Machala, Ecuador

Autor para correspondencia: secorrea_est@utmachala.edu.ec.

Fecha de recepción: 06 de Septiembre de 2016 - Fecha de aceptación: 20 de diciembre de 2016

\section{Resumen}

Estructurar competencias en la gestión administrativa de una empresa implica optimizar el funcionamiento de la misma. Las instituciones que las han implementado han logrado resultados alineados con las políticas institucionales y han verificado que implementar procedimientos que gestionen las competencias de los trabajadores permite mejorar la calidad en el desempeño de los mismos y a la empresa crecer en el mercado. En las exportadoras de banano del cantón Machala, la gestión por competencias es efímera; de allí la necesidad de aplicar procesos que verifiquen las habilidades y destrezas de los diferentes equipos de trabajo que conforman una empresa para lograr un desempeño eficiente. Es prioridad que estas instituciones se encuentren implementando cualidades competitivas para volverse eficientes y enfrentar la competencia de mejor manera; así también, se requiere la incorporación de programas formativos que anexen procesos de formación, reconocimiento de competencias y evaluación de desempeño para buscar la certificación empresarial que los convertirá en líderes.

Palabras claves: Competencias; gestión por competencias; crecimiento empresarial; exportadoras de banano; talento humano

\begin{abstract}
Structuring skills in administrative management of a company involves optimizing the operation thereof. The institutions that have implemented have managed aligned with institutional policies and results have verified that implement procedures to manage the skills of workers to improve the quality in the performance of them and the company to grow in the market. Banana exporters in the canton Machala, competency management is fleeting; hence the need for processes to verify the skills of the different teams that make up a company to achieve an efficient performance. Priority is that these institutions are implementing competitive qualities to become efficient and meet the competition better; well, incorporating training programs that annexed formation processes, recognition of skills and performance evaluation to seek certification that business leaders become required.
\end{abstract}

Key Words: competence; competence management; business growth; export banana; human talent 


\section{Introducción}

En los años noventa la noción de competencia adquirió un notable protagonismo en el léxico de numerosas disciplinas tales como la sociología, las ciencias de la educación, la psicología y la administración de empresas. La utilización del término competencia en el campo empresarial se remonta a los primeros de la psicología diferencial de los años 60, no obstante se rescató como vocablo característico de los recursos humanos en la década de los ochenta y se generalizó posteriormente, en los noventa (McClelland, 1973).

En 1981 en el Reino Unido, se aplicó técnicas de análisis como: “Critica Incidente Tecnique" orientada a definir habilidades básicas que debería tener el personal de las organizaciones para garantizar un desenvolvimiento adecuado. En 1986 un grupo de expertos se une a éste, utilizando para ello técnicas activas, para medir habilidades requeridas en los diferentes cargos, logrando como hecho la unión entre el Departamento de Recursos Humanos con las demás áreas de la organización en el concepto de eficacias. En España en 1988 se presentó un proyecto sobre las habilidades que las organizaciones deberían tener en cuenta para garantizar desempeños laborales exitosos. Desde entonces se cambió el término "Habilidades" por "Competencias" y se inicia su difusión con mucho éxito (Gallego, 2000).

En los países desarrollados, la gestión por competencias en las empresas ha tenido un amplio progreso, porque sus gobiernos se han interesado en crear incentivos políticos y económicos, abrir créditos sin mayor burocracia, crear metas de exportación para pequeños y medianos productores, además de contar con una fuerza laboral especializada, en función del reto que enfrenta el nuevo modelo económico (López Luna y Chaparro Maldonado, 2006).

Las empresas en el Ecuador actualmente ponen énfasis en el desarrollo del talento humano, aplicando nuevas técnicas que conllevan a la búsqueda de estrategias, que permitan fomentar los conocimientos del personal, capacitando y evaluando sus habilidades y destrezas, para lograr un mayor rendimiento que beneficie a las organizaciones, de tal manera que se encuentren preparados para enfrentar así el mercado donde la competencia cumple un papel muy importante (Rubio y García, 2014).

Las competencias de los funcionarios y trabajadores se traducen en lo cognitivo a las habilidades y destrezas para un desempeño eficiente en el campo laboral; de hecho hoy en día, en la era del conocimiento y el avance tecnológico, de forma vertiginosa se constituye prioritario, que el ser humano se capacite y cuente con cualidades competitivas para ser eficiente; de ahí el interés de conocer qué repercusión tiene en el campo empresarial las competencias del personal en el desarrollo económico (Mejía Giraldo, Bravo Castillo y Montoya Serrano, 2013).

El objetivo de la presente investigación se enfoca en determinar la incidencia de la gestión por competencias en el crecimiento empresarial de las exportadoras de banano de la ciudad de Machala, ya que constituyen la base de la economía de la región y la generadora de divisas en las exportaciones. Uno de los principales limitantes que se ostentó al realizar el levantamiento de la información, es que no se logró establecer una comunicación directa con los jefes de talento humano de las empresas antes mencionadas, ya que no todas las organizaciones poseen en su estructura un departamento responsable para dirigir y controlar al personal. 


\section{Desarrollo}

\section{Definición de Competencias:}

Las competencias laborales según Martha Alles (2006) hacen referencia a características esenciales que poseen las personas, lo cual permite que desempeñen sus funciones de forma diferenciada y eficaz en su puesto de trabajo. Mientras que la Organización Internacional de Trabajo (como se citó en Segovia, 2012, p.25-30) manifiesta que es "una capacidad efectiva para llevar a cabo exitosamente una actividad laboral plenamente identificada. La competencia laboral no es una probabilidad de éxito en la ejecución de un trabajo; es una capacidad real y demostrada".

Para Beneitone (como se citó en Manríquez Pantoja, 2012) las competencias son fundamentales para que el ser humano se desenvuelva eficientemente en su diario vivir, y a su vez pueda ser competitivo en un ambiente cambiante y en constante evolución. En comparación con Pérez (como se citó en Pérez Capdevila 2012), afirma que la única manera de que las personas obtengan un desenvolvimiento adecuado es conociendo con que aptitudes cuenta y con qué actitudes desarrolla sus tareas cotidianas.

"Las competencias son consecuencia de la integración en forma coherente de las actitudes (visión, misión, filosofía); aptitudes (rutinas, políticas, aprendizaje); capacidades (liderazgo, habilidades, experiencias); que le permiten interactuar interna y extremamente de forma eficiente, favoreciendo su adaptación y facilitando el desempeño de la competencias tecnológicas y personales" (González Martínez, 2000, p.42).

Lichtenberger (como se citó en Massó y Lozares, 2012) demuestra que el saber, saberhacer, saber-estar forman parte de mezcla de conocimientos, aptitudes y experiencia que inherentemente son visibles durante el desarrollo de las personas de acuerdo al entorno en que este se encuentre y por ende en un contorno en específico a la competencia no se la puede evidenciar notablemente más que en la aplicación de la misma ya que depende mucho en donde, como y con qué herramientas está siendo ejecutada.

En relación con Lichtenberger, (Manriquez Pantoja, 2012) demuestra en la figura 1, cuatro fases sobre el proceso para establecer que una competencia esta normalmente desarrollada. Los primeros procesos, son base en una competencia, muestra una clara interacción con lo cognitivo y los siguientes procesos con la conducta.

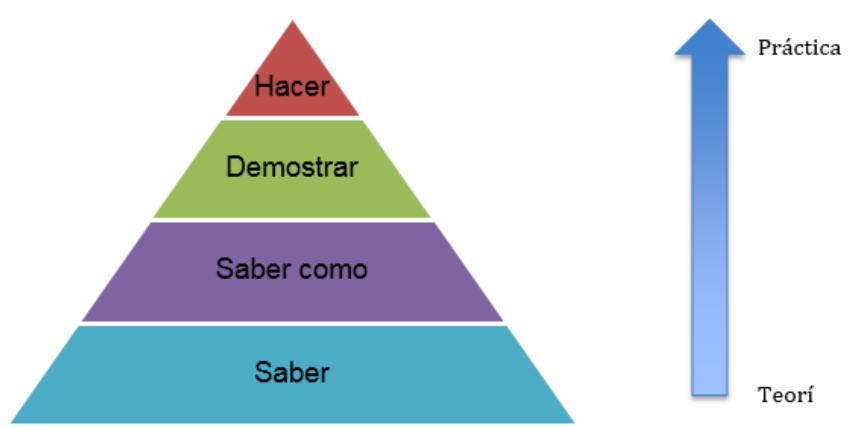

Figura I. Adaptado de Miller (Como se citó en Pantoja, 2012) 
En contexto con lo resaltado anteriormente, las empresas tienen la necesidad de promover la gestión del conocimiento y aplicar un modelo de competencia laborales, en el cual la mayoría de sus integrantes conocerán a fondo su perfil de cargo y adecuado a las actividades en el puesto al que desempeña, el propósito es adaptar un perfil basado en competencias de acuerdo a lo que la organización requiera para la consecución de sus objetivos (Sandoval Sucre y Pernalete Chirinos, 2014).

\section{Gestión por competencias:}

La gestión por competencia se define como una herramienta del talento humano con una propuesta específica basada en los resultados de los empleados en un determinado proceso de gestión y a su vez alineado sinérgicamente con los resultados de la empresa. Todo esto se enmarca bajo instrumentos de evaluación e identificación de competencias (Guerrero Dávalos, Valverde Aparicio, y Tatiana Gorjup, 2013).

Por tanto, Santos (citado en Chávez Hernández, 2012) asegura que la importancia de la gestión de competencia está fundamentada bajo criterios que conducen a una gestión del personal adecuada basada en las competencias que aporten a la consecución de los objetivos organizacionales.

La gestión de competencias en el ámbito empresarial, hace énfasis a un compromiso de cada uno de sus miembros, lo cual, propone un sistema eficiente en dirección a la capacidad innovadora y creativa bajo el marco social-laboral y empresarial en donde se desempeña, siempre y cuando este fundamentada en las habilidades del personal (García González, García Rodríguez, Pérez Marimón, González Pérez, y Martínez López, 2014).

En definitiva, su aplicación en las diferentes organizaciones ha cobrado un valor importante en cuanto a los requerimientos del mercado, tratando de desarrollar e implementar un personal idóneo a las necesidades, es así que se transforma en una tarea ardua la selección del talento humano, en lo que se refiere a competencias y conocimientos, logrando con esto una ubicación correcta en sus respectivas áreas de cada miembro de la organización (Chávez Hernández, 2012).

El modelo propicio para la gestión de competencia debe conformarse por las siguientes partes: 


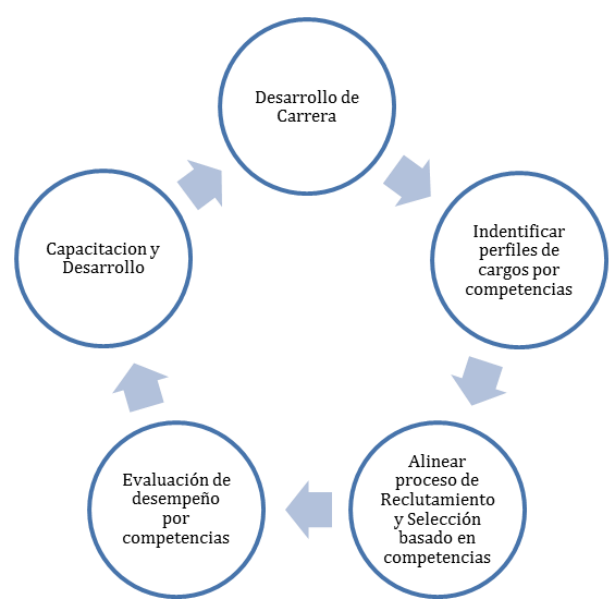

Figura 2. Adaptado de (Sandoval Sucre y Pernalete Chirinos, 2014)

Para Delgado (como se citó García Dousat, Reyes Jardinez, y Javier Carballo, 2009), la implementación de un modelo de gestión por competencias supone entre otras cosas, un cambio cultural en cuanto a cómo las organizaciones evalúan el conocimiento (lo capta selecciona, organiza, distingue y presenta) y le da prioridad a aprender de su propia pericia y a focalizarse en obtener, recopilar y utilizar el discernimiento para resolver dificultades y desarrollar la inteligencia y adaptabilidad de la empresa.

Para Tejada Fernández y Navío Gámez (2005) aplicar un modelo de gestión por competencia en las empresas brinda un sin número de ventajas de entre las cuales se resalta las siguientes:

- Resaltar al talento humano como clave del desarrollo organizacional y el tiempo de vida de la misma.

- Pasar de funciones y puestos muy delimitados a una gestión integrada de procesos y trabajo en equipo.

- Colaborar bajo el modelo de competencias a que sus empleados se adapten rápidamente a los cambios permanentes que se dan en el mercado competitivo.

- Fomentar una mentalidad de constante aprendizaje.

- Brindar a los integrantes de la organización nuevos conocimientos aplicables en recompensa de su dedicación y esfuerzo.

¿Contar con una adecuada gestión de competencias fomentará el crecimiento empresarial? Tal como lo manifiestan, Dorta y Verona (2006), el crecimiento empresarial depende de diferentes factores que pueden ser internos como la edad y el tamaño, la motivación, la estructura de propiedad y la gestión del conocimiento, y entre los factores externos se pueden mencionar algunos relativos al entorno sectorial (competidores, clientes y proveedores), y otros de nivel superior o macro entorno.

No obstante, Rojo y González (2007) afirma que las necesidades que surgen en las empresas, con respecto a la supervivencias en el mercado se refieren estas en el factor de 
competencias que ha tratado siempre de mantenerse y de ser reconocidas. Basando su apoyo para tal situación al crecimiento interno y externo de la compañía.

Por ende la gestión por competencia se postula como una herramienta clave para que las empresas crezcan y se adapten a cambios que se presenten en el entorno, mediante la adecuada dirección del talento humano (García Dousat, Reyes Jardinez, y Javier Carballo, 2009).

\section{Métodos de Investigación:}

\section{Metodología}

Para la presente investigación se usó el método cuantitativo, el cual según Ugalde y Balbastre (2013) explicó que se da como un requisito de comprender la profundidad de los hechos que ocurren a su alrededor para el comportamiento del ser humano, se basa en pequeñas muestras que se dirigen en un tema puntual y se necesita del razonamiento para obtener conclusiones que se manifiesta de los hechos aceptados como válidos.

Además, se utilizó el método exploratorio que se desarrolla cuando existe un tema o problema de investigación que ha tenido poco estudio por lo que no resulta posible realizar una generalización de los resultados a otros grupos con características semejantes (Hernández y Castro, 2013).

\section{Datos de la muestra:}

El presente estudio de investigación se realizó a 28 profesionales, representantes de las exportadoras de banano del Cantón Machala, información obtenida mediante un oficio emitido por parte del Ministerio de Agricultura, Ganadería, Acuacultura y Pesca (MAGAP).

\section{Descripción de variables:}

Se utilizó como variables independientes: la gestión por competencia, la edad de la empresa y el personal que cuenta con título universitario, la cual nos permitió conocer si son factores que inciden en la variable dependiente que es el crecimiento empresarial.

\section{Técnica de Investigación:}

La técnica de investigación que se utilizó fue la encuesta, ya que Casas, Repullo y Donado (2003) manifiestan que es un instrumento el cual permite recopilar información de forma ágil y segura.

Se adaptó de una encuesta sobre gestión por competencias, realizada en Bogotá Colombia por la consultora internacional Martha Alles, en su revista técnica virtual por competencias.

Para este instrumento se elaboró 5 preguntas abiertas de factores internos que permiten medir el crecimiento empresarial como son la edad de la empresa, gasto anual por formación del personal, gasto anual en salarios, número de empleados y cuantos tienen título universitarios, se 
incluyó 7 preguntas de escala nominal la cual afirma Coronado Padilla (2007) es la más primordial y la forma más elemental de medir. Se relaciona a las unidades de estudio (objetos, personas, etc.) basándose en una o más características, con dos categorías de Si o No las cuales se denominan dicotómicas, para determinar si las empresas exportadoras de banano aplican la gestión por competencias.

\section{Método estadístico}

Para obtener los resultados del objetivo planteado, se utilizó el programa Stata 19 que es un programa estadístico muy eficaz para analizar, manipular e interpretar gráficamente datos (Rochina Barrachina y Sanchis LLopis, 2002). En el cual se implementó un modelo de regresión lineal múltiple, "en este modelo la variable de respuesta se puede relacionar con variables regresoras. Se justifica aplicar el modelo de regresión lineal múltiple cuando interviene más de una variable independiente". (Astorga, 2014, p.236)

$$
Y=\beta_{0}+\beta_{1} X+\beta_{2} X_{2}+\ldots+\beta_{K} X_{K}+\varepsilon
$$

\section{Hipótesis:}

H1: La gestión por competencias es uno de los factores principales que incide en el crecimiento empresarial de las exportadoras de banano.

$\mathrm{H} 2$ : Existen otros factores que son parte del crecimiento empresarial de las exportadoras de banano.

\section{Resultados}

Los resultados que se obtuvieron en la investigación indican en la Tabla 1 una relación favorable y altamente significativa $(\mathrm{p}<0,00)$, entre el crecimiento empresarial y la gestión por competencia, por consiguiente las exportadoras de banano, diseñan perfiles de competencias para cada puesto de trabajo, planes estratégicos para el desarrollo y administración de competencias, realizan evaluaciones del potencial por competencias al personal, implementan programas de capacitación y entrenamiento por competencias e incentivan al personal en base a sus conocimientos, habilidades y cualidades, por lo tanto aplican la gestión por competencias, lo cual reflejan un crecimiento empresarial eficiente, corroborando así lo planteado en la primera hipótesis.

Referente a la segunda hipótesis se confirma que existen otros factores internos que inciden en el crecimiento empresarial de las exportadoras de banano, estos son la edad de la empresa, $(\mathrm{p}<0,00)$ y el que cuente con personal profesional $(\mathrm{p}<0,02)$. Sin embargo en su gran mayoría estas empresas que desarrollan este tipo de actividad poseen un número mayoritario de empleados que no cuentan con título profesional, es decir sus competencias y habilidades son fundamentales para que la empresa se desarrolle en el mercado competitivo.

\section{Tabla 1: Resultado de la regresión}




\begin{tabular}{cc}
\hline & Coef. \\
\hline Crecimiento Empresarial & \\
\hline Gestión por competencia & 2,968072 \\
\hline Edad de la Empresa & $(8,10)$ \\
\hline Personal profesional & 0,932901 \\
\hline & $(4,25)$ \\
\hline R-squared & 1,167816 \\
\hline F (3,24) & $(3,40)$ \\
\hline
\end{tabular}

\section{Conclusiones}

El presente estudio concluye en que la aplicación de la gestión por competencia en las empresas exportadoras de banano que se encuentran ubicadas en el cantón Machala, ha tenido un efecto positivo en el talento humano, pues se ha verificado una mejora en sus capacidades y habilidades, lo cual ha sido considerado como prioridad para el desarrollo empresarial y económico, utilizando métodos y técnicas que conlleven a cumplir con los objetivos de la empresa.

Pese a que la gestión por competencias es una herramienta que pocos conocen y utilizan, en la actualidad ha servido de gran apoyo para las organizaciones, como referente para la selección y ubicación de su personal en áreas estratégicas que ayuden a su emprendimiento y desarrollo.

Por consiguiente las empresas que han aplicado la gestión de competencias han presentado un mayor crecimiento en sus ventas y han logrado obtener una mejor rentabilidad, lo cual es el objetivo de toda organización.

\section{Bibliografía}

Alles, M. A. (2006). Dirección estratégica de Recursos Humanos: gestión por competencias. En Dirección estratégica de Recursos Humanos: gestión por competencias (Vol. 1). Buenos Aires: Ediciones Granica S.A. Recuperado el 27 de Agosto de 2016

Alles, M. A. (2006). Selección por competencias. En Selección por competencias. Ediciones Granica S.A. Recuperado el 11 de Agosto de 2016

Alonso Suárez, C., Parra Rojas, R., \& Arronte Cruz, L. (2010). Un sistema de gestión del capital humano en la EMCE basado en las competencias laborales. Ingeniería Industrial, XXXI (1), 1-8. Obtenido de http://www.redalyc.org/articulo.oa?id=360433572003 
Astorga Gómez, J. M. (Marzo de 2014). Aplicación de modelos de regresión lineal para determinar las armónicas de tensión y corriente. Ingeniería Energética, 35(3), 234-241. Obtenido de http://scielo.sld.cu/scielo.php?script=sci_arttext\&pid=S1815-59012014000300008

Blásquez Santana, F., Dorta Velásquez, J. A., \& Verona Martel, M. C. (Enero de 2006). Concepto, perspectivas y medida del crecimiento empresarial. Cuadernos de Administración, 165195. Obtenido de http://www.redalyc.org/articulo.oa?id=20503107

Casas Anguita, J., Repullo Labradora, J. R., \& Donado Campos, J. (2003). La encuesta como técnica de investigación. Elaboración de cuestionarios y tratamiento estadístico de los datos.

Atención Primaria, 31(8), 527-538. Doi: 10.1016/S0212-6567(03)70728-8

Chávez Hernández, N. (2012). La gestión por competencias y ejercicio del coaching empresarial, dos estrategias internas para la organización. Pensamiento \& Gestión (33), 140-161. Obtenido de http://www.redalyc.org/articulo.oa?id=64624867007

Coronado Padilla, J. (Julio-Diciembre de 2007). Escalas de Medición. Paradigmas, 2(2), 104-125. Obtenido de https://dialnet.unirioja.es/descarga/articulo/4942056.pdf

Gallego, M. (Julio de 2000). Gestión humana basada en competencias contribución efectiva al logro de objetivos organizacionales. Revista Universidad EAFIT, 36(11), 63-71. Obtenido de http://www.redalyc.org/pdf/215/21511906.pdf

García Dousat, Y., Reyes Jardinez, L., \& Javier Carballo, C. (Abril de 2009). ¿Por qué la importancia de implementar Sistemas de Gestión por Competencias en nuestras organizaciones? Ciencias Holguín, XV (2), 1-9. Obtenido de http://www.redalyc.org/articulo.oa?id=181517990005

García González, M., García Rodríguez, A., Pérez Marimón, R. A., González Pérez, M., \& Martínez López, Y. (2014). La gestión por competencias para el desempeño exitoso, análisis desde el sector empresarial cubano. Revista científica avances, 16(1), 64-75. Obtenido de https://dialnet.unirioja.es/servlet/articulo?codigo=5350913

González Martínez, M. (Enero-Marzo de 2000). De la gestión por competencias a la gestión por competencias personales, hacia un nuevo modelo de gestión de personas en la era del conocimiento. Ciencia y Sociedad, XXV (1), 35-61. Obtenido de http://www.redalyc.org/articulo.oa?id=87011348002

Guerrero Dávalos, C., Valverde Aparicio, M., \& Tatiana Gorjup, M. (2013). Un análisis de la gestión por competencias en la empresa española. De la teoría a la práctica. Contaduría y Administración, 58(1), 251-288. Obtenido de http://www.redalyc.org/articulo.oa?id=39525580010 
Hernández, K., \& Castro, A. M. (23 de 04 de 2013). Un estudio exploratorio para caracterizar las prácticas de la lectura. Actualidades de la investigación en educación, 13(2), 1-17. Obtenido de http://revistas.ucr.ac.cr/index.php/aie/article/view/11730/18203: http://www.redalyc.org/articulo.oa?id=44727049009

López Luna, E., \& Chaparro Maldonado, M. Y. (Julio-Diciembre de 2006). Competencias laborales del trabajador vistas desde el mercado laboral. Tabula Rasa(5), 261-275. Obtenido de http://www.redalyc.org/articulo.oa?id=39600514

Manríquez Pantoja, L. (2012). ¿Evaluación en competencias? Estudios pedagógicos, XXXVIII (1), 367-380. Obtenido de http://www.redalyc.org/articulo.oa?id=173524158023

Massó, M., \& Lozares, C. (2012). Un análisis de la gestión de las competencias en el trabajo: el caso de los mandos intermedios de una factoría de carrocería y montaje de automóviles. Cuadernos de Relaciones Laborales, 30(1), 211-233. doi:10.5209/rev_CRLA.2012.v30.n1.39121

McClelland, D. C. (1 de January de 1973). Testing for competence rather than for "intelligence." American Psychologis, 28(1), 1-14. Doi: 10.1037/h0034092

Mejía Giraldo, A., Bravo Castillo, M., \& Montoya Serrano, A. (2013). El factor del talento humano en las organizaciones. Ingeniería Industrial, 34(1), 2-11. Obtenido de http://scielo.sld.cu/scielo.php?script=sci_arttext\&pid=S1815-59362013000100002

Pérez Capdevilav, J. (2012). Medición de competencias e impacto monetario sobre el pago del Salario. Ciencia en su PC (2), 115-116. Obtenido de http://www.redalyc.org/articulo.oa?id=181324082009

Rochina Barrachina, M. E., \& Sanchis LLopis, J. (2002). Stata 7.0 para windows. Revista de Economía Aplicada, X (28), 163-174. Obtenido de http://www.redalyc.org/articulo.oa?id=96917634008

Rojo, M., \& González, G. (2007). El crecimiento empresarial vía cooperaciones entre compañías. Estudio Empírico sobre el sector turístico español. Investigaciones Europeas de Dirección y Economía de la Empresa, 13(3), 127-145. Obtenido de http://www.aedemvirtual.com/articulos/iedee/v13/133127.pdf

Rubio, P., \& García, J. (Septiembre de 2014). Gestión de la formación por competencias en operarios de alimentación como ventaja competitiva de comedores industriales en empresas privadas. Ciencias de Investigación de Ciencias Administrativas y gerenciales, 12(1), 207-230. Obtenido de https://dialnet.unirioja.es/descarga/articulo/5028157.pdf

Sandoval Sucre, F., \& Pernalete Chirinos, D. (2014). Marco de trabajo para gestionar las competencias laborales. Revista Venezolana de Información, Tecnología y Conocimiento, 11(2), 11-32. Obtenido de http://www.redalyc.org/articulo.oa?id=82332932002 
Segovia Díaz, S. C. (2012). Modelo de gestión por competencias aplicado al proceso de capacitación en una institución de salud pública de la quinta región de Chile. Boletín Científico

Sapiens $\quad$ Research, 2(1), 23-30. Obtenido de https://dialnet.unirioja.es/servlet/articulo?codigo=3841401

Tejada Fernández, J., \& Navío Gámez, A. (2005). El desarrollo y la gestión de competencias profesionales: una mirada desde la formación. Revista Iberoamericana de Educación, 2-15. Obtenido de rieoei.org/delos lectores/1089Tejada.pdf

Ugalde Binda, N., \& Balbastre Venavent, F. (2013). Investigación cuantitativa e investigación cualitativa: buscando las ventajas de las diferentes metodologías de investigación. Ciencias Económicas, $\quad 31(2), \quad 179-187 . \quad$ Obtenido de https://dialnet.unirioja.es/servlet/articulo?codigo $=4512073$ 